\title{
Does universal 16S rRNA gene amplicon sequencing of environmental communities provide an accurate description of nitrifying guilds?
}

\author{
Diwan, Vaibhav; Albrechtsen, Hans-Jørgen; Smets, Barth F.; Dechesne, Arnaud
}

Published in:

Journal of Microbiological Methods

Link to article, DOI:

10.1016/j.mimet.2018.05.025

Publication date:

2018

Document Version

Peer reviewed version

Link back to DTU Orbit

Citation (APA):

Diwan, V., Albrechtsen, H-J., Smets, B. F., \& Dechesne, A. (2018). Does universal 16S rRNA gene amplicon sequencing of environmental communities provide an accurate description of nitrifying guilds? Journal of Microbiological Methods, 151, 28-34. https://doi.org/10.1016/j.mimet.2018.05.025

\section{General rights}

Copyright and moral rights for the publications made accessible in the public portal are retained by the authors and/or other copyright owners and it is a condition of accessing publications that users recognise and abide by the legal requirements associated with these rights.

- Users may download and print one copy of any publication from the public portal for the purpose of private study or research.

- You may not further distribute the material or use it for any profit-making activity or commercial gain

- You may freely distribute the URL identifying the publication in the public portal 
1 Does universal 16S rRNA gene amplicon sequencing of environmental communities provide an

2 accurate description of nitrifying guilds?

3

4 Vaibhav Diwan, Hans-Jørgen Albrechtsen, Barth F. Smets, Arnaud Dechesne*

5 Department of Environmental Engineering, Technical University of Denmark, Miljoevej, 2800 Kgs, Lyngby, Denmark

$6 \quad$ Vaibhav Diwan, e-mail: vdiw@env.dtu.dk

$7 \quad$ Hans-Jørgen Albrechtsen, e-mail: hana@env.dtu.dk

$8 \quad$ Barth F. Smets, e-mail: bfsm@env.dtu.dk

$9 *$ Corresponding author:

10 Arnaud Dechesne

11 Address: Department of Environmental Engineering, Technical University of Denmark, Building 115, Miljoevej, 2800 Kgs,

12 Lyngby, Denmark

13 E-mail: arde@env.dtu.dk

14 Telephone: +45 45252291

15 Fax: +4545932850 


\section{Abstract}

23 Universal (i.e., targeting most bacteria/prokaryotes) 16S rRNA gene based amplicon sequencing is 24 widely used for assessing microbial communities due to its low cost, time efficiency, and ability to 25 provide a full overview of the community. However, it is currently unclear if it can yield reliable 26 information on specific microbial guilds, which can be obtained by using primer sets targeting functional genes or specific16S rRNA gene sequences. Here, we compared the relative abundance, diversity, richness, and composition of selected guilds (nitrifiers), obtained from universal 16S rRNA gene based amplicon sequencing and from guild targeted approaches. The universal amplicon sequencing provided 1) accurate estimates of nitrifier composition, 2) clustering of the samples based on these compositions consistent with sample origin, 3) estimates of the relative abundance of the guilds correlated with those obtained from the targeted approaches and within $\sim 1.2$ orders of magnitude of them, but with measurable bias that should be considered when comparing estimates from both approaches. In contrast, the diversity and richness estimations using the universal 16S rRNA based amplicon sequencing were likely limited by the sequencing depth; therefore, we suggest preferring targeted approaches for assessing nitrifiers diversity and richness or using sequencing depth larger than those currently typically practiced.

Keywords: Diversity; Drinking water treatment; Wastewater treatment; Nitrifiers; AOB; Nitrospira. 
44 Community-wide high throughput amplicon sequencing of one or multiple hypervariable regions of the 16S rRNA gene has become a routine tool to describe the composition and diversity of microbial communities (Bartram et al., 2011; Caporaso et al., 2011). Beyond such an overall community assessment, it is often desirable to quantify and characterize specific constituent guilds in terms of abundance, composition, and diversity. For many guilds, the 16S rRNA gene can be informative because phylogenetic conservation of functional traits is common (Philippot et al., 2010; Martiny et al., 2013). In fact, if the microbial guild of interest consists of one or a few monophyletic clades (for example, ammonia-, nitrite-, or methane-oxidizers), it is possible to utilize 16S rRNA gene primers to specifically target the guild (Degrange and Bardin 1995; Kowalchuk et al., 1997; Hermansson \& Lindgren, 2001; Graham et al., 2007). Additionally, if conserved signature functional genes exist, targeting them can be a strategy. For example, amoA, which codes for a subunit of the ammonia monooxygenase can be used to target ammonia oxidizing prokaryotes; and $n x r B$, which codes for the beta subunit of the nitrite oxidoreductase can be used to target nitrite oxidizing bacteria (NOB) (Norton, 2011; Pester et al., 2013). Targeting functional genes can be advantageous to access withinguild diversity, due to their high rate of evolution compared to the 16S rRNA gene (Dopheide et al. 59 2015). In contrast to a non-specific approach such as universal (i.e., designed to target most bacteria) 16S rRNA gene amplicon sequencing, any guild-specific approach provides information only on the targeted guild but none on the rest of the community, which is an obvious limitation (Xue et al. 2013; 
64 microbial community, but provide only limited information on non-dominant guilds because of their

65 low contribution to the total sequence pool.

66 Therefore, it is currently unclear whether universal 16S rRNA gene amplicon sequencing is sufficient

67 to obtain reliable information on specific microbial guilds and, more precisely, whether it correctly

68 differentiates between samples with high and low guild diversity and whether it provides sufficient

69 compositional information to identify samples with similar guild composition.

70 An additional concern regarding microbial community analysis using amplicon sequencing lies in its

71 ability to provide reliable estimates of the relative abundance of the community constituents. Indeed,

72 the quantitative nature of amplicon sequencing has been questioned (Zhou et al. 2011). Studies using

73 mock communities have shown that the results from 16S rRNA gene based amplicon can be biased

74 based on choice of primers and at different in-vitro (DNA extraction) and in-silico (sequencing and taxonomic assignment) experimental stages (Brooks et al. 2015; Parada et al. 2016; Thijs et al. 2017).

76 Despite known biases, 16S rRNA gene based amplicon sequencing has been used successfully for 77 quantitative assessment of the ubiquitous taxa in the bacterial community (Ibarbalz et al. 2014). 78 However, to our knowledge, the literature provides no assessment of the reliability of universal amplicon sequencing for quantifying specific guilds. Therefore, one of the aims of this study was to fill 80 this gap.

81 Here, using nitrifiers as model guilds, we compared bacterial 16S rRNA gene amplicon sequencing 82 (further referred to as 'universal approach') to a guild-targeted approach in their abilities to infer 83 relative abundance, diversity, richness, and composition. Nitrifying microbial guilds are a key to 84 ammonium oxidation in many natural and engineered ecosystems. They were traditionally strictly 85 divided into ammonia-oxidizing prokaryotes and nitrite oxidizing bacteria (NOB) before the recent 
discovery of Nitrospira types that can fully oxidize ammonia (Comammox; Daims et al. 2015; van Kessel et al., 2015; Palomo et al. 2016). To target key nitrifying guilds, we used the functional genes amoA for ammonia oxidizing bacteria (AOB) and archaea (AOA); and $n x r B$ for the main NOB genera Nitrospira and Nitrobacter (Arp and Stein 2003; Francis, Beman, and Kuypers 2007; Vanparys et al., 2007; Pester et al., 2013)

\section{Material and Methods}

\subsection{Biomass sampling and DNA extraction}

The biomass originated from the top $(0-10 \mathrm{~cm})$ layer of the after filter (AF) of 4 drinking water treatment plants (DWTP) in Denmark and from the nitrifying reactor (NR) of wastewater treatment plants (WWTP) from Denmark and Sweden and from an anammox reactor (Sjolunda (AR)) in a WWTP from Sweden (Figure S1). Three samples for each plant were used for qPCR. For the 16S rRNA gene amplicon sequencing, five samples were analyzed for three DWTP (Glostrup, Hillerod, and Odense (AF)) and single samples for all other plants.

DNA was extracted from $0.5 \mathrm{~g}$ of wet drained sand from DWTP and $0.5 \mathrm{~g}$ sludge from WWTP using FastDNA $^{\mathrm{TM}}$ spin kit for soil (MP Biomedicals, Solon, OH, USA) according to manufacturer's instructions. Duplicate DNA extractions were done for each sample. DNA concentration was estimated using NanoDrop ND-1000 UV-VIS Spectrophotometer (Thermo Fisher Scientific, Waltham, MA, USA) and the extracts were stored at $-20^{\circ} \mathrm{C}$ until further analysis.

\section{2 qPCR, PCR and amplicon sequencing}

Quantitative PCR (qPCR) analyses were conducted on a Chromo4 thermocycler using Opticon Monitor version 3 (BioRad). Each qPCR reaction contained $12.5 \mu \mathrm{L}$ of $2 \mathrm{X}$ iQ SYBR green Supermix (Bio-Rad 
107 Laboratories, Hercules, California, United States), 500 mM primer, DNA template (10 ng), and 108 DNA/RNA-free water (Qiagen, Hilden, Germany) to 25 $\mu$ L. For each sample, DNA with average 109 concentration $\sim 26$ and $\sim 68 \mathrm{ng} / \mu \mathrm{l}$ for sand and sludge samples respectively was sent for 16S rRNA 110 gene, amoA, and $n x r B$ PCR, purification and amplicon sequencing ( $2 \times 300$ nucleotides) using the

111 Illumina MiSeq platform at the DTU Multi Assay Core Center (Kgs. Lyngby, DK). For the universal 112 16S rRNA gene sequencing, primers (Bakt_341F and Bakt_805R, spanning the V3-V4 regions) and 113 PCR conditions were from Herlemann et al., 2011. For amoA AOB sequencing and qPCR, primers 114 (amoA-1F and amoA-2R primers) and PCR conditions were from Rotthauwe et. al., 1997. For $n \times r B$ 115 Nitrospira sequencing and qPCR, primers (nxrB169f and nxrB638r primers) and PCR conditions were 116 from Pester et al., 2013. Primers (CTO189fA-B, CTO189fC, and RT1r) and PCR conditions for AOB 117 16S rRNA gene qPCR were from Kowalchuk et al., 1997. QPCR efficiencies and correlation 118 coefficients obtained from the standard curves are in Table S1.

\subsection{Bioinformatics analysis}

Sequences generated as paired FASTQ files were processed using DADA2 (Version - 1.4; Callahan et al., 2016). DADA2 was preferred over other clustering-based methods as it recovers sequence variants that can vary by as little as a single nucleotide and thus avoids aggregation of sequences at arbitrary identity cutoff. DADA2 was used for quality filtering, trimming, de-replicating the reads, for inferring sequence variation through default error model parameters, for merging paired reads, for removing chimera, and for assigning taxonomy using Silva reference database v123 for 16S rRNA gene and custom taxonomy files for $a m o A$ and $n x r B$. A very similar pipeline was used for $a m o A$ and $n x r B$, except that for amoA only the forward reads (237 bp after trimming for quality) were analyzed due to the amplicon length. We applied frameshift correction (from package DECIPHER version 2.6; Wright 
129 2016) and discarded sequences that were too distant from our reference sequences. the data obtained

130 from DADA2 was analyzed using Phyloseq R Package (Version - 1.7.12; McMurdie and Holmes

131 2013). Raw sequence files were deposited into the sequence read archive at GenBank under the study

132 accession number SRP127282.

\subsection{Relative abundance estimation of AOB and Nitrospira}

134 Universal 16S rRNA gene amplicon sequencing data was used to perform CaRcone analysis to obtain

135 the average 16S rRNA gene copies per genome in the amplicon libraries (R script

136 https://github.com/ardagulay/CaRcone---Community-average-rRNA-gene-copy-nr-estimator). The

137 total numbers of bacteria per gram of biomass were derived by normalizing the gene copy number from

138 qPCR with 1.75 16S rRNA gene copies per genome obtained from the CaRcone analysis. The abundance of AOB and Nitrospira cells per gram of biomass was obtained by assuming 2 amoA and 2 nxrB copies per genome (McTavish, Fuchs, and Hooper 1993; Lücker et al. 2010). Thus, comparing $n x r B$ for Nitrospira and amoA for AOB with 16S rRNA gene was feasible as the abundance values

142 obtained from two genes were normalized per genome.

143 The relative fractions of AOB and Nitrospira were calculated by dividing the normalized abundance

144 estimates obtained from amoA and $n x r B$ based qPCR by that obtained from 16S rRNA gene based total

145 bacteria qPCR. Similarly, AOB and Nitrospira abundance estimates obtained from amoA and $n \times r B$

146 based sequencing were compared to their respective values obtained after dividing reads assigned to

147 AOB and Nitrospira in the 16S rRNA gene based total bacteria sequencing by the total number of 148 reads. 


\subsection{Statistical analysis}

151

152

153

154

155

156

157

158

159

160

161

162

163

164

165

166

167

168

169

170

The alpha diversity metrics, Shannon diversity and observed richness were calculated using the ‘estimate_richness’ function in the Phyloseq R package version (Version - 1.7.12; McMurdie and Holmes, 2013). The estimates of Shannon diversity after rarefaction were calculated by performing rarefaction using the minimal number of sequences in each gene library, as we would lose a large amount of sequences from amoA and $n x r B$ libraries if we rarified to the minimum number of sequences assigned to AOB and Nitrospira from the 16S rRNA gene library. For phylogenetic diversity calculation, first the sequences were aligned using the 'AlignSeqs' function in the DECIPHER R package (Version 2.0.2; Wright, 2015), then a neighbor joining (NJ) tree was constructed using phangorn R package (Version 2.3.1; Schliep, 2011), phylogenetic diversity (Faith's PD; the sum of the branches of the phylogenetic tree) was then calculated using the PhyloMeasures R package (Version 2.1; Faith, 1992; Tsirogiannis and Sandel, 2016).

To statistically compare the relative abundance and diversity estimates from universal and targeted approaches we used major axis (MA) regression (a Type II regression model) because for both type of estimates none of the variables ( $\mathrm{x}$ and $\mathrm{y}$ ) were experimentally controlled (Pierre Legendre and Legendre 1988). As the relative abundance estimates were homoscedastic on the log scale, we performed the regression analysis on the log scale with average abundance values from each plant. The MA regression was performed using ‘lmodel2’ package version 1.7-3 in R (P Legendre 2018).

To evaluate whether the universal and targeted approaches separated the samples similarly based on nitrifier composition, Principal Coordinate Analysis (PCoA) of samples based on their nitrifier composition obtained from universal and targeted approaches was performed separately. The PCoA 
ordination matrix based on Bray Curtis similarities was generated using 'ordinate' function and the PCoA plot was generated using ‘plot_ordination’ function in the Phyloseq R package ( Version -

In the universal approach, sequences assigned to nitrifier guilds were extracted to estimate the relative

1.7.12; McMurdie and Holmes, 2013). Further, Procrustes analysis was performed to compare the multidimensional shapes of the two PCoAs by transforming them into a state of best superimposition (Peres-Neto and Jackson 2001). The significance of the similarity of the two PCoA matrices was then tested by a procrustean randomized test (PROTEST) that determines whether the sum of the residual nonconformities was less than that expected by chance (Jackson, 1995).

The clustering analysis was performed using the K-medoids clustering, a partitioning method that clusters a set of objects into K clusters, with K set based on a priori knowledge (Jin and Han 2010). K medoids clustering method was applied using PAM clustering in vegan (version 2.4-3) and cluster (version 2.0.6) R packages (Dixon 2003; Mächler et al., 2012). The Clustering analysis figures were generated using ‘clusplot’ function in cluster R package (version 2.0.6; Mächler et al., 2012) and further edited using Inkscape (version 0.921; Bah, 2007).

\section{Results and discussion}

We investigated biomass extracted from three nitrifying and one anammox wastewater treatment bioreactors and from four biological rapid sand filters producing potable water (Figure S1). At both types of plants, nitrifiers perform a fundamental role in ammonium removal and are known to range from $1 \%$ to $10 \%$ in abundance relative to the whole microbial community (Wagner et al., 2002; Gülay et al., 2016; Tatari et al., 2017).

abundance, diversity, richness, and composition of nitrifying guilds (Table S2). These estimates were 
192 then compared to those obtained from the guild-targeted approaches (qPCR for relative abundance and amplicon sequencing for diversity and composition; Table S2).

194 Nitrobacter (NOB) and ammonia-oxidizing archaea (AOA) were minor fractions of the nitrifiers in the

195 analyzed communities. Indeed, both types of nitrifiers were undetected in the universal approach; the amplification of Nitrobacter $n \times r B$ prior to amplicon sequencing failed; and AOA were below or close to the detection limit of the amoA qPCR assay (10 gene copies per reaction) in WWTP and DWTP, respectively (data not shown). Therefore, here we focus only on AOB and Nitrospira; further collectively referred to as nitrifiers.

In the universal approach, 20,372 and 346,879 sequences were assigned to AOB and Nitrospira, respectively, across all sampling sites. From the targeted approach, 501,381 sequences were assigned to AOB (amoA) and 2,165,787 to Nitrospira (nxrB) (Table S3).

\subsection{Relative abundance of nitrifiers}

Targeted quantification of AOB was performed by qPCR using two primer sets targeting either betaproteobacterial-AOB-specific 16S rRNA gene or amoA. The relative abundances obtained from the universal approach were always lower than the 16S rRNA gene based qPCR (Figure 1A) but higher than the amoA qPCR (Figure S2-A). These results are consistent with previous observations from environments similar to those investigated in this study indicating that the $16 \mathrm{~S}$ rRNA gene AOB primers from Kowalchuk et al., 1997, being unspecific, tend to overestimate AOB abundance, and that the amoA primers can underestimate AOB abundance depending upon the AOB composition (Figure

211 S2-B; Dechesne et al. 2016; Tatari et al., 2017). Nitrifier composition for this study is presented and 212 discussed in further sections. Compared to the universal approach, the $n x r B$ qPCR yielded slightly 213 higher relative abundance estimates for Nitrospira in all WWTP but lower for all DWTP (Figure 1B). 
214 These observations for DWTP are consistent with previous findings of Nirospira relative abundance

215 comparison of 16S rRNA gene based qPCR specific to Nitrospira and universal amplicon sequencing

216 (Gülay et al. 2016). Overall, for both of the guilds, even if we observed inconsitstency between the

217 targeted and the universal approaches, the universal approach based estimates were always within $\sim 1.2$

218 orders of magnitude of the targeted approaches.

219 Using MA regression analysis, we explored next whether the estimates from both approaches were

220 linearly related. For AOB, the slope was significantly different from 0 (p value 0.003 ) and very close to

2211 (slope estimate $=0.9$ ), suggesting a direct proportionnality between the approaches. However, the

222 intercept was negative ( $\mathrm{p}$ value $<0.05$ ), consistent with the observation made erlier that the 16S rRNA

223 gene based AOB quantification by qPCR tend to be significantly higher than the one based on universal

224 amplicon sequencing (Figure 1-A). For Nitrospira, the intercept was not statistically different from 0

225 and the slope was significantly higher than 1 (slope estimate $=2.2$ ) suggesting that the Nitrospira

226 quantification based on universal amplicon sequencing increases more rapidly than $n x r B$ qPCR with

227 Nitrospira relative abundance (Figure 1-B).

228 Overall, the estimates of relative abundance of both guilds (AOB and Nitrospira) from universal 229 approach correlated with those obatined from the targeted approaches and were within 1.2 orders of 230 magnitude of them, but with measurable bias. The universal approach certainly provides useful

231 quantitative information for AOB and Nitrospira but the highlighted biases should be considered when

232 comparing estimates from universal and targeted approaches for these guilds. 

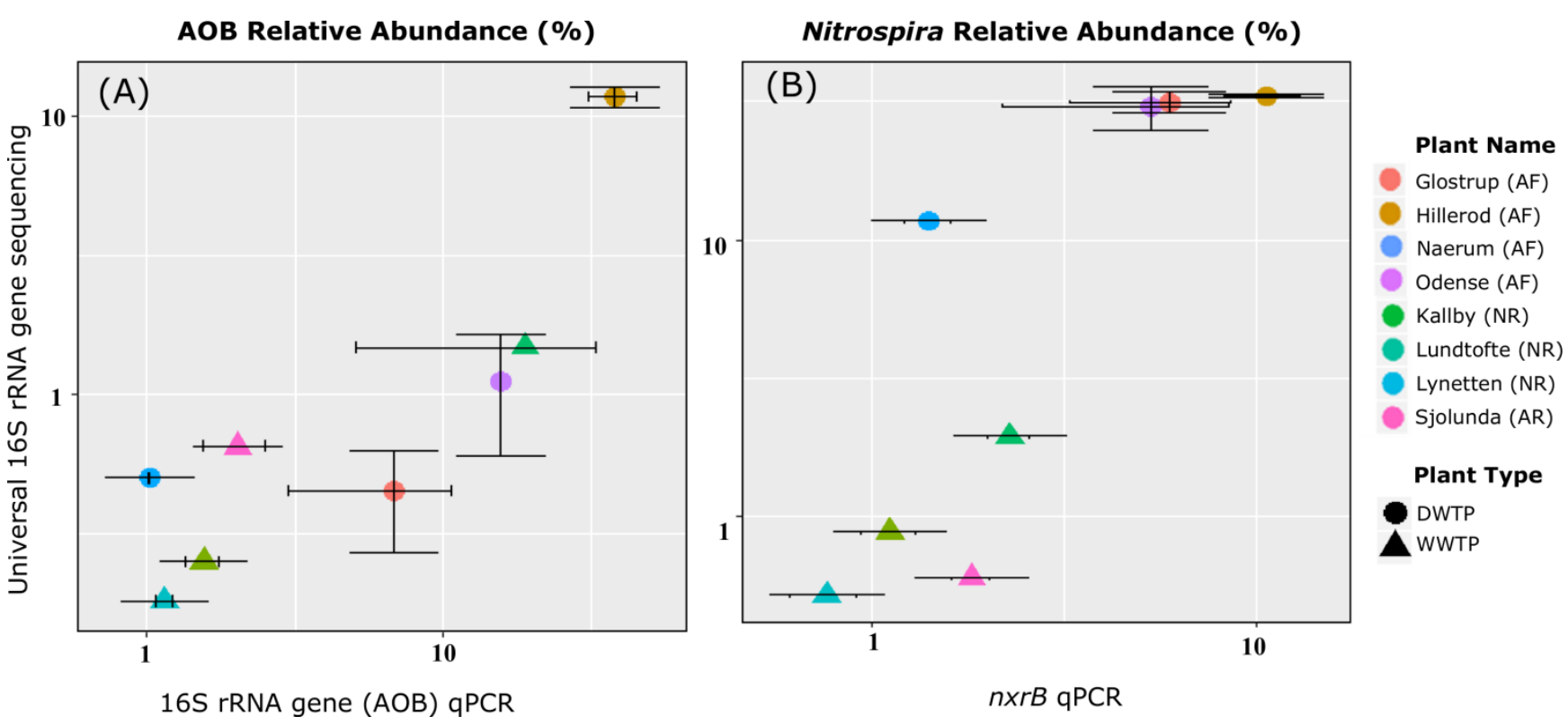

Figure 1 (color in print): Comparison of the relative abundance of AOB (A) and Nitrospira (B) based

on universal (y-axis) and targeted (x-axis) approach. Universal 16S rRNA gene based amplicon

sequencing was compared with qPCR (target: 16S rRNA gene for AOB in panel A and $n x r B$ for

Nitrospira in panel B) for four after filters (AF) from DWTP, three nitrifying reactors (NR) and one

anammox reactor from WWTP. The error bars represent the standard deviation for each plant.

\subsection{Alpha diversity of nitrifiers}

240 For both guilds and irrespective of the metric used, the targeted approach always resulted in higher

241 alpha diversity values compared to the universal approach (Figure 2). We ascribe this observation to

242 the facts that the functional gene libraries contained approximately 24 fold (AOB) and $\sim 6$ fold

243 (Nitrospira) more sequences than retrieved from the universal sequencing (Table S3) and that diversity

244 estimates can be highly reliant on the depth of sequencing (number of sequences per sample; Caporaso

245 et al., 2011; Gihring et al., 2012; Smith and Peay, 2014). Additionally, the rate of evolution of

246 functional genes ( $a m o A, n x r B, r p o B$ etc.) is known to be higher than that of ribosomal genes (16S 
247 rRNA gene), which makes them more phylogenetically resolutive ( Case et al., 2007; Pester et al.,

248 2012; Pester et al., 2013). Thus, using the targeted approach on functional genes results in the detection

249 of more sequence variants than with the universal approach, which translates to higher but also, likely

250 more accurate, diversity estimates.

251 For both guilds, the Shannon diversity and observed richness calculated from the universal approach

252 correlated very poorly with that estimated from the targeted approach (Figure $2 \mathrm{~A}, \mathrm{~B}, \mathrm{D}$ ). This was also

253 true when these indexes were calculated after rarefaction to the minimal number of sequences in each

254 comparison (Figures S3 and S4). Phylogenetic diversity (Faith's PD; the sum of the branches of the

255 phylogenetic tree) for AOB and observed richness (without rarefaction) for Nitrospira were the only

256 diversity indices with a significantly positive correlation between both approaches ( $p$ value 0.009 ;

257 Figure 2C and p value 0,01; Figure 2E). This frequent inconsistency between targeted and universal

258 approach suggests that the guild-targeted approaches should be preferred to estimate the diversity of

259 AOB and Nitrospira. 

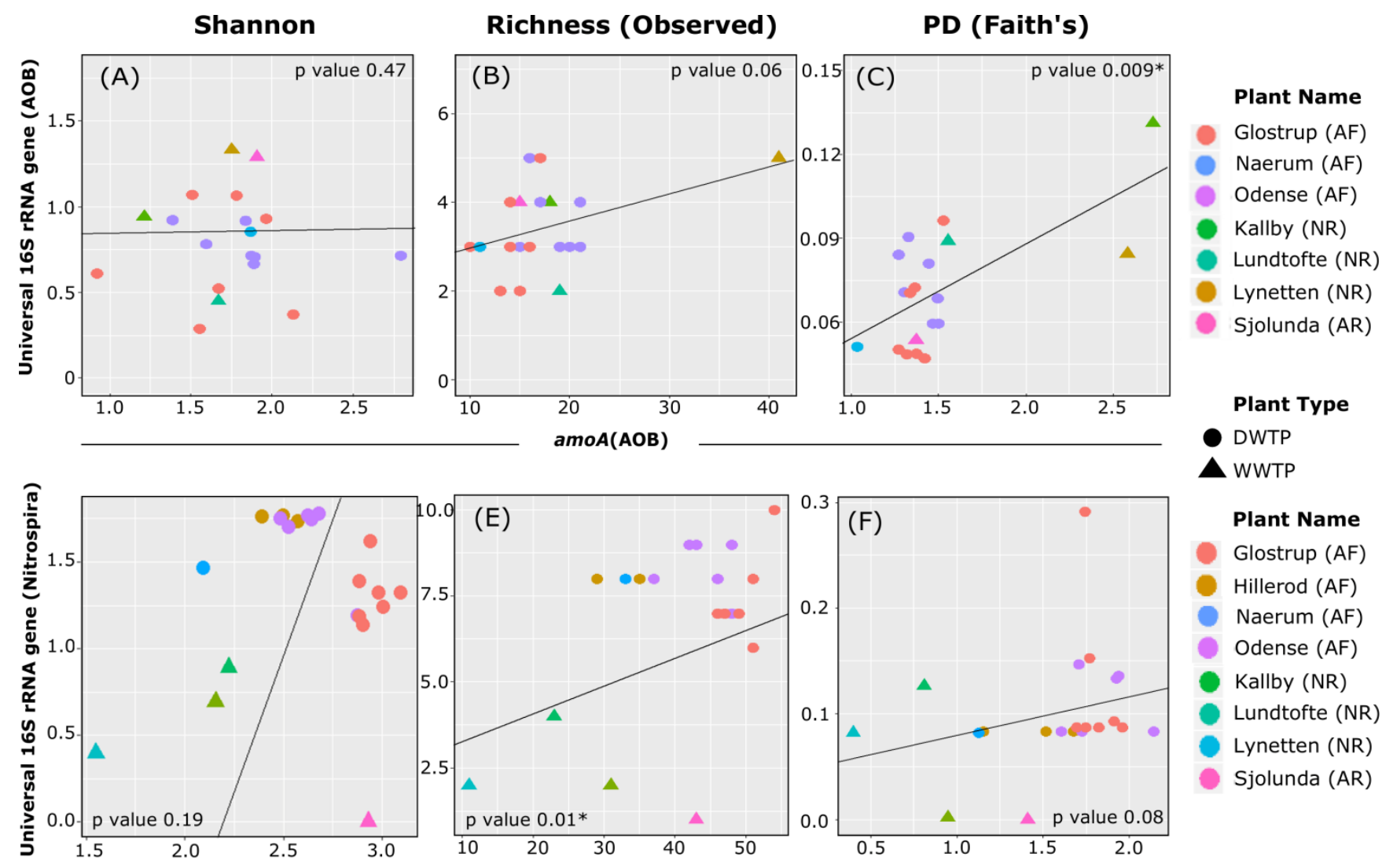

Plant Name

Glostrup (AF)

Hillerod (AF)

Naerum (AF)

Odense (AF)

Kallby (NR)

Lundtofte (NR)

Lynetten (NR)

Sjolunda (AR)

nxrB (Nitrospira)

261 Figure 2 (color in print): Comparison of alpha diversity (Shannon; A and D), observed richness (B and E) and phylogenetic diversity (Faith’s PD; C and F) of AOB and Nitrospira based on universal (16S rRNA gene) and targeted (amoA -top and $n x r B$-bottom) approaches. Scales on $\mathrm{x}$ and $\mathrm{y}$-axis are different. The black line is the major axis regression and the respective p values are mentioned in each panel suffixed with a star sign if significant. For Nitrospira, a sample for which zero values were obtained with the universal approach (Sjolunda (AR)) was excluded from the linear regression. Likewise, for one DWTP, no valid AOB amoA sequences were obtained (Table S3). 


\subsection{Composition of nitrifiers}

\subsubsection{AOB composition}

273 The universal approach only yielded sequences assigned to cluster 6 (represented by Nitrosomonas 274 aestuarii and Nitrosomonas marina) and 7 (represented by Nitrosomonas europaea and Nitrosomonas 275 eutropha strains) whereas the targeted approach also detected clusters 0, 2, 3, (Nitrosospira) and 8 276 (represented by Nitrosomonas nitrosa; Figure S5). Both approaches identified the same dominant 277 cluster (Cluster 6) in five out of seven sites. Only for one plant (Sjolunda) did both approaches provide 278 the exact same picture of the cluster-level composition. For all DWTP and one WWTP, the universal 279 approach only showed the presence of cluster 6; whereas the targeted approach indicated the presence of additional clusters: cluster 7 in all these plants, plus cluster 0, 2, 3 and 8 in one WWTP (Kallby; (Figure S5). These observations relate with the low relative abundance of AOB observed earlier by universal 16S rRNA gene sequencing (Figure 1A) as clusters other than cluster 6 were largely unmapped for four out of seven sites by the universal approach due to their very low relative fraction to the total community.

Finally, at the subcluster taxonomic resolution, we found that all samples where cluster 6 was predominant consisted mainly of subcluster 6A members (represented by Nitrosomonas oligotropha and Nitrosomonas sp. ls79A3; Figure S6). As mentioned in previous studies, amoA AOB based primers (from Rotthauwe et al., 1997), having been designed primarily from cluster 7 members sequences, underestimate AOB abundance when subcluster 6A members are dominant (Dechesne et al., 2016). 290 This likely explains why amoA based qPCR underestimated AOB in these plants (Figure S2). 


\subsubsection{Nitrospira composition}

294 Nitrospira composition inferred from both approaches was largely similar for all sites (Figure S7). 295 Both approaches identified strong compositional differences between DWTP and WWTP, the former 296 being dominated by lineage 2 and the later by lineage 1 (Figure S7).

297 Overall, the universal approach successfully identified the dominant clusters/lineages within AOB and 298 Nitrospira for most plants. Therefore, the universal approach can be a preferred choice for estimating 299 the composition of these guilds, especially when the focus is on dominant guild members.

\subsection{Samples ordination and clustering based on nitrifiers composition}

301

302

303

304

In the PCoA analysis, both approaches separated DWTP and WWTP samples for both the guilds (Figure 3) but the PCoA plots obtained from the targeted approaches were visually different from that generated from their universal approach counterpart (Figure 3). Procrustean randomized test (PROTEST) was not significant for both guilds (AOB: $\mathrm{p}$ value $=0.8$; Nitrospira: $\mathrm{p}$ value $=0.5$ ), confirming the visual differences as significant.

Next, K-medoids clustering was performed to evaluate how the two approaches grouped (clustered) the samples based on their nitrifier composition. Here, we set $\mathrm{K}$ as the number of plants because we assumed that samples originating from the same plant should cluster together because communities from similar ecological sites tend to be similar in composition (Whittaker 1965). 

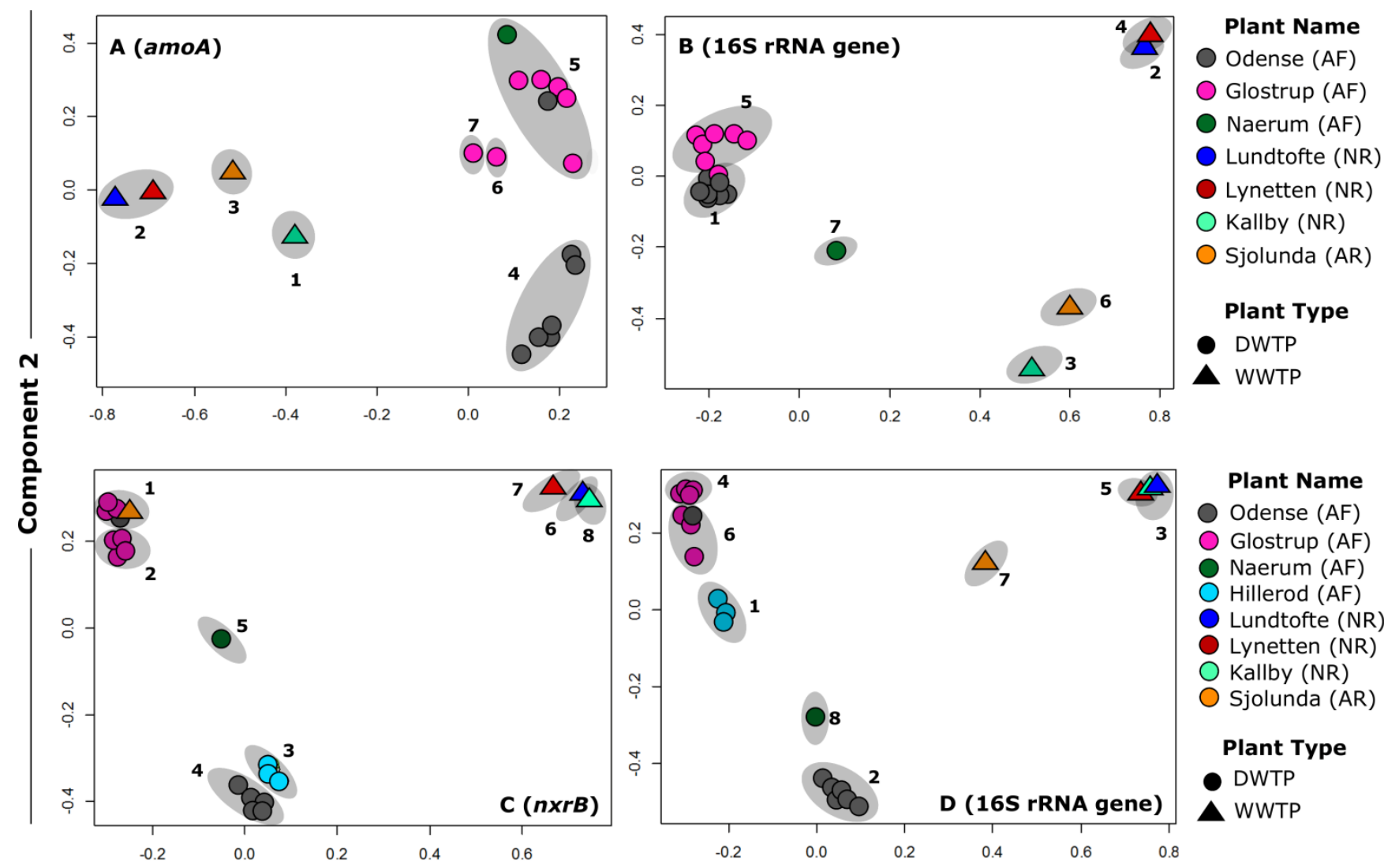

Plant Type

- DWTP

- WWTP

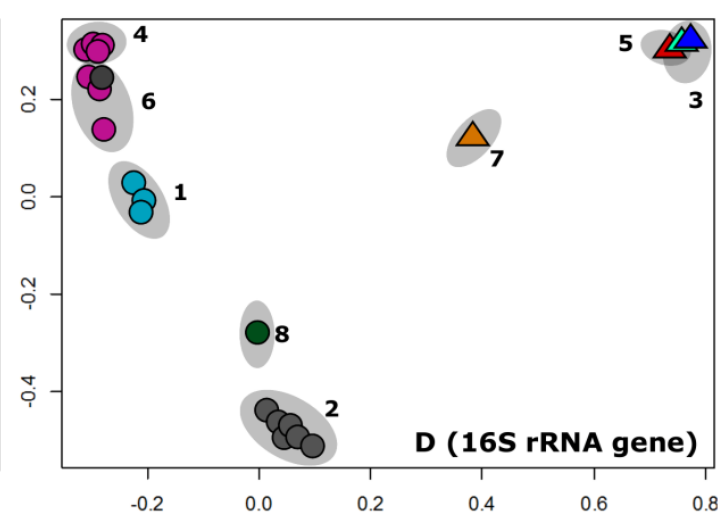

Plant Name

Odense (AF)

Glostrup (AF)

Naerum (AF)

$\bigcirc$ Hillerod (AF)

Lundtofte (NR)

Lynetten (NR)

Kallby (NR)

$\bigcirc$ Sjolunda (AR)

Plant Type

- DWTP

WWTP

Component 1

314 Figure 3 (color in print): Clustering analysis of samples after PCoA ordination of their compositions

315 of AOB (panels A \& B) and Nitrospira (panels C \& D) obtained from the targeted and universal

316 approach. Shaded ellipses followed by cluster number represent the clusters. Each solid circle and

317 triangle is a sample, with color coding according to the plant. Seven replicate samples were used for

318 Odense and Glostrup, three for Hillerod and one for all other plants. For AOB, the two components

319 explain 50.4\% of the variability for the PCoA based on amoA (A) and 57.3\% for 16S rRNA gene (B).

320 For Nitrospira the two components explain $67.5 \%$ of the variability for the PCoA based on $n x r B(C)$

321 and $71.3 \%$ for $16 \mathrm{~S}$ rRNA gene (D). The number of clusters (K) were adjusted to 7 and 8 for AOB and

322 Nitrospira, respectively, according to the number of plants sampled. 


\subsubsection{Sample clustering based on AOB composition}

325 The universal approach separated the samples into seven clusters corresponding to the seven plants, 326 except for one replicate which was misplaced (Figure 3B). amoA incorrectly distributed the DWTP 327 samples into four clusters, with one cluster comprising samples from all three plants (Figure 3A). 328 Samples from two WWTP were placed in the same cluster (Cluster 2; figure 3A) whereas they were separated by the 16S rRNA gene (Cluster 2 and 4; figure 3B). Therefore, it is apparent that the targeted approach did not provide better clustering than the universal approach.

\subsubsection{Sample clustering based on Nitrospira composition}

The universal and targeted approaches for Nitrospira clustered samples similarly with some minor variations (Figure $3 \mathrm{C}$ and D). For example, $n x r B$ based analysis separated all plants except for one cluster which contained samples from two DWTP and one WWTP (Cluster 1; figure 3C). For the universal approach, all plants were separated except for one sample from a DWTP that clustered with samples from another DWTP (Cluster 6; figure 3D). Two WWTP that were separated (Cluster 6 and 8; figure 3C) by the targeted approach were now clustered together (Cluster 3; figure 3D). Here, the universal and the targeted approaches thus gave similar clustering outputs.

\section{Conclusion}

Taken together, our results provide the first systematic validation of the use of universal approaches for quantification and composition estimation of nitrifying guilds, as done by several authors (Rughöft et al., 2016; Ramanathan et al. 2017; Meerbergen et al., 2017; Saarenheimo et al. 2017). Overall, the $16 \mathrm{~S}$ rRNA gene based universal amplicon sequencing of environmental communities can be efficiently used as substitute to more narrow targeted approaches for:

1) The assessment of nitrifier composition, as it captured the main ecological signal (the most dominant taxa) for all plants. 
2) Sample clustering based on nitrifier composition, as it correctly separated samples from different plants.

349 The universal approach also provided relative abundance estimates within $\sim 1.2$ orders of magnitude of 350 those from the targeted approaches, but with systematic biases that should be considered especially 351 when comparing quantification estimates from both approaches.

352 Lastly, for the universal approach, diversity estimation was likely limited by the sequencing depth.

353 Therefore, we suggest preferring targeted approaches for assessing nitrifiers diversity, unless much 354 higher sequencing depths than commonly currently practiced are used.

\section{Conflicts of interest}

356 The authors declare no conflicts of interest.

\section{Acknowledgments}

358 We would like to thank Florian B. Wagner, Elena Torresi and Sara Ekström for assisting with sample 359 collection. We also thank the plant operators at the Odense, Glostrup, Hillerod, Naerum DWTP and 360 Lundtofte, Lynetten, Kally and Sjolunda WWTP for their cooperation.

\section{Funding:}

362 This research was financially supported by MERMAID (an initial training network funded by the People Programme - Marie Sklodowska-Curie Actions- of the European Union's Seventh Framework 364 Programme FP7/2007-2013/ under REA grant agreement no. 1607492). 
370 Arp, Daniel J., and Lisa Y. Stein. 2003. “Metabolism of Inorganic N Compounds by Ammonia-

371 Oxidizing Bacteria.” Critical Reviews in Biochemistry and Molecular Biology 38 (6): 471-95. doi:10.1080/10409230390267446.

Bah, Tavmjong. 2007. Inkscape: Guide to a Vector Drawing Program. First. Upper Saddle River, NJ, USA: Prentice Hall Press.

Callahan, Benjamin J, Paul J McMurdie, Michael J Rosen, Andrew W Han, Amy Jo A Johnson, and Susan P Holmes. 2016. “DADA2: High-Resolution Sample Inference from Illumina Amplicon Data.” Nat Meth 13 (7): 581-83. http://dx.doi.org/10.1038/nmeth.3869. 
Caporaso, J Gregory, Christian L Lauber, William A Walters, Donna Berg-Lyons, Catherine A Lozupone, Peter J Turnbaugh, Noah Fierer, and Rob Knight. 2011. “Global Patterns of 16S RRNA Diversity at a Depth of Millions of Sequences per Sample.” Proceedings of the National Academy of Sciences of the United States of America 108 Suppl (Supplement_1): 4516-22. doi:10.1073/pnas.1000080107.

Daims, H., E.V. Lebedeva, P. Pjevac, P. Han, C. Herbold, M. Albertsen, N. Jehmlich, et al. 2015. “Complete Nitrification by Nitrospira Bacteria.” Nature 528 (7583). doi:10.1038/nature16461.

Dechesne, Arnaud, Sanin Musovic, Alejandro Palomo, Vaibhav Diwan, and Barth F. Smets. 2016. "Underestimation of Ammonia-Oxidizing Bacteria Abundance by Amplification Bias in AmoATargeted QPCR.” Microbial Biotechnology 9 (4): 519-24. doi:10.1111/1751-7915.12366.

Degrange, V., and R. Bardin. 1995. "Detection and Counting of Nitrobacter Populations in Soil by PCR.” Applied and Environmental Microbiology 61 (6): 2093-98.

Dixon, Philip. 2003. “VEGAN, a Package of \{R\} Functions for Community Ecology.” J Veg Sci 2003 14 (6): 927-30.

Dopheide, Andrew, Gavin Lear, Zhili He, Jizhong Zhou, and Gillian D. Lewis. 2015. "Functional Gene Composition, Diversity and Redundancy in Microbial Stream Biofilm Communities.” PLoS ONE 10 (4): 1-21. doi:10.1371/journal.pone.0123179.

Faith, D P. 1992. “Conservation Evaluation and Phylogenetic Diversity.” Biological Conservation 61: 1-10. doi:10.1890/0012-9658(2006)87[1465:ATTFHF]2.0.CO;2.

Francis, Christopher A., J. Michael Beman, and M. M M Kuypers. 2007. “New Processes and Players 
in the Nitrogen Cycle: The Microbial Ecology of Anaerobic and Archaeal Ammonia Oxidation.” ISME Journal 1 (1): 19-27. doi:10.1038/ismej.2007.8.

411 Gihring, Thomas M., Stefan J. Green, and Christopher W. Schadt. 2012. “Massively Parallel RRNA

Graham, David W, Charles W Knapp, Erik S Van Vleck, Katie Bloor, Teresa B Lane, and Christopher E Graham. 2007. "Experimental Demonstration of Chaotic Instability in Biological Nitrification.” The ISME Journal 1 (5): 385-93. doi:10.1038/ismej.2007.45.

Gülay, Arda, Sanin Musovic, Hans-Jørgen Albrechtsen, Waleed Abu Al-Soud, Søren J Sørensen, and Barth F Smets. 2016. “Ecological Patterns, Diversity and Core Taxa of Microbial Communities in Groundwater-Fed Rapid Gravity Filters.” The ISME Journal 10 (9): 2209-22. doi:10.1038/ismej.2016.16.

Herlemann, Daniel P.R., Matthias Labrenz, Klaus Jürgens, Stefan Bertilsson, Joanna J Waniek, and Anders F Andersson. 2011. “Transitions in Bacterial Communities along the $2000 \mathrm{Km}$ Salinity Gradient of the Baltic Sea.” ISME Journal 5 (10): 1571-79. doi:10.1038/ismej.2011.41.

Hermansson, Anna, and Per-eric Lindgren. 2001. "Quantification of Ammonia-Oxidizing Bacteria in Arable Soil by Real-Time PCR” 67 (2): 972-76. doi:10.1128/AEM.67.2.972.

Ibarbalz, Federico M., María Victoria Pérez, Eva L M Figuerola, and Leonardo Erijman. 2014. “The Bias Associated with Amplicon Sequencing Does Not Affect the Quantitative Assessment of 
Bacterial Community Dynamics.” PLoS ONE 9 (6). doi:10.1371/journal.pone.0099722.

430 Jackson, Donald A. 1995. “PROTEST: A PROcrustean Randomization TEST of Community

431 Environment Concordance.” Écoscience 2 (3): 297-303.

432 Jin, Xin, and Jiawei Han. 2010. “K-Medoids Clustering.” In Encyclopedia of Machine Learning, edited 433 by Claude Sammut and Geoffrey I Webb, 564-65. Boston, MA: Springer US. doi:10.1007/978-0434 387-30164-8_426.

435 Kowalchuk, G A, J R Stephen, W De Boer, J I Prosser, T M Embley, and J W Woldendorp. 1997.

436 “Analysis of Ammonia-Oxidizing Bacteria of the Beta Subdivision of the Class Proteobacteria in

437 Coastal Sand Dunes by Denaturing Gradient Gel Electrophoresis and Sequencing of PCR-

438 Amplified 16S Ribosomal DNA Fragments.” Applied and Environmental Microbiology 63 (4): 439 1489-97. doi:10.1128/AEM.67.10.4880.

440 Legendre, P. 2018. “Lmodel2: Model II Regression.” R Package Version 1.7-3. https://cran.r$441 \quad$ project.org/web/packages/lmodel2/index.html.

442 Legendre, Pierre, and Louis Legendre. 1988. "Numerical Ecology, Volume 24.” (Developments in $443 \quad$ Environmental Modelling) 24: 504. doi:10.1017/CBO9781107415324.004.

444 Liu, Zongzhi, Catherine Lozupone, Micah Hamady, Frederic D. Bushman, and Rob Knight. 2007. 445 “Short Pyrosequencing Reads Suffice for Accurate Microbial Community Analysis.” Nucleic $446 \quad$ Acids Research 35 (18). doi:10.1093/nar/gkm541.

447 Lücker, Sebastian, Michael Wagner, Frank Maixner, Eric Pelletier, Hanna Koch, Benoit Vacherie, 448 Thomas Rattei, et al. 2010. “A Nitrospira Metagenome Illuminates the Physiology and Evolution 
of Globally Important Nitrite-Oxidizing Bacteria.” Proceedings of the National Academy of Sciences of the United States of America 107 (30): 13479-84. doi:10.1073/pnas.1003860107.

451

452

453

454

455

Mächler, Martin, Peter Rousseeuw, Anja Struyf, Mia Hubert, and Kurt Hornik. 2012. Cluster: Cluster Analysis Basics and Extensions. R Packages. Vol. 1.

Martiny, Adam C, Kathleen Treseder, and Gordon Pusch. 2013. "Phylogenetic Conservatism of Functional Traits in Microorganisms.” The ISME Journal 7 (4). Nature Publishing Group: 830-38. doi:10.1038/ismej.2012.160.

McMurdie, Paul J, and Susan Holmes. 2013. “Phyloseq: An R Package for Reproducible Interactive Analysis and Graphics of Microbiome Census Data.” PLOS ONE 8 (4). Public Library of Science: e61217. https://doi.org/10.1371/journal.pone.0061217.

McTavish, Hugh, James a. Fuchs, and Alan B. Hooper. 1993. "Sequence of the Gene Coding for Ammonia Monooxygenaseไnin Nitrosomonas Europaea.” Journal of Bacteriology 175 (8): 243644. doi:citeulike-article-id:10421008.

Meerbergen, Ken, Maarten Van Geel, Michael Waud, Kris A. Willems, Raf Dewil, Jan Van Impe, Lise Appels, and Bart Lievens. 2017. “Assessing the Composition of Microbial Communities in Textile Wastewater Treatment Plants in Comparison with Municipal Wastewater Treatment Plants.” MicrobiologyOpen 6 (1): 1-13. doi:10.1002/mbo3.413.

Norton, Jeanette M. 2011. “Diversity and Environmental Distribution of Ammonia-Oxidizing Bacteria.” In Nitrification, edited by Martin G. Ward, Bess B. and Arp, Daniel J. and Klotz, 3955. doi:doi:10.1128/9781555817145.ch3. 
Palomo, Alejandro, S Jane Fowler, Arda Gülay, Simon Rasmussen, Thomas Sicheritz-Ponten, and Barth F Smets. 2016. “Metagenomic Analysis of Rapid Gravity Sand Filter Microbial Communities Suggests Novel Physiology of Nitrospira Spp.” The ISME Journal 10 (11): 2569_ 
Ramanathan, Bhargavi, Andrew M. Boddicker, Timberley M. Roane, and Annika C. Mosier. 2017.

494 "Nitrifier Gene Abundance and Diversity in Sediments Impacted by Acid Mine Drainage.” Frontiers in Microbiology 8 (November): 1-16. doi:10.3389/fmicb.2017.02136.

\section{6}

Rotthauwe, J. H., Witzel, K. P., and Liesack, W. 1997. “The Ammonia Monooxygenase Structural Gene AmoA as a Functional Marker : Molecular Fine-Scale Analysis of Natural AmmoniaOxidizing Populations.” Applied and Environmental Microbiology 63 (12): 4704-12.

Rotthauwe, J H, K P Witzel, and W Liesack. 1997. “The Ammonia Monooxygenase Structural Gene AmoA as a Functional Marker: Molecular Fine-Scale Analysis of Natural Ammonia-Oxidizing Populations.” Applied and Environmental Microbiology 63 (12). American Society for Microbiology: 4704-12.

Rughöft, Saskia, Martina Herrmann, Cassandre S. Lazar, Simone Cesarz, Shaun R. Levick, Susan E. Trumbore, and Kirsten Küsel. 2016. “Corrigendum: Community Composition and Abundance of Bacterial, Archaeal, and Nitrifying Populations in Savanna Soils on Contrasting Bedrock Material in Kruger National Park, South Africa [Front. Microbiol, (2016), 7, (1638)]. Doi: 10.3389/Fmicb.2016.01638.” Frontiers in Microbiology 7 (NOV): 1-16. doi:10.3389/fmicb.2016.01954.

Saarenheimo, Jatta, Sanni L. Aalto, Antti J. Rissanen, and Marja Tiirola. 2017. "Microbial Community Response on Wastewater Discharge in Boreal Lake Sediments.” Frontiers in Microbiology 8 
(APR): 1-12. doi:10.3389/fmicb.2017.00750.

512 Schliep, Klaus Peter. 2011. “Phangorn: Phylogenetic Analysis in R.” Bioinformatics 27 (4): $592-93$.

513 doi:10.1093/bioinformatics/btq706.

514 Smith, Dylan P., and Kabir G. Peay. 2014. "Sequence Depth, Not PCR Replication, Improves

515 Ecological Inference from next Generation DNA Sequencing.” PLoS ONE 9 (2).

516 doi:10.1371/journal.pone.0090234.

517 Tatari, Karolina, Sanin Musovic, Arda Gülay, Arnaud Dechesne, Hans-Jørgen Albrechtsen, and Barth

518 F. Smets. 2017. "Density and Distribution of Nitrifying Guilds in Rapid Sand Filters for Drinking

519 Water Production: Dominance of Nitrospira Spp.” Water Research 127. Elsevier Ltd: 239-48.

$520 \quad$ doi:10.1016/j.watres.2017.10.023.

521 Thijs, Sofie, Michiel Op De Beeck, Bram Beckers, Sascha Truyens, Vincent Stevens, Jonathan D. Van

522 Hamme, Nele Weyens, and Jaco Vangronsveld. 2017. “Comparative Evaluation of Four Bacteria-

523 Specific Primer Pairs for 16S RRNA Gene Surveys.” Frontiers in Microbiology 8 (MAR): 1-15.

524 doi:10.3389/fmicb.2017.00494.

525 Tsirogiannis, Constantinos, and Brody Sandel. 2016. “PhyloMeasures: A Package for Computing

526 Phylogenetic Biodiversity Measures and Their Statistical Moments.” Ecography 39 (7): 709-14.

527 doi:10.1111/ecog.01814.

528 van Kessel, Maartje A H J, Daan R Speth, Mads Albertsen, Per H Nielsen, Huub J M Op den Camp,

529 Boran Kartal, Mike S M Jetten, and Sebastian Lücker. 2015. “Complete Nitrification by a Single

530 Microorganism.” Nature 528 (7583). Nature Publishing Group: 555-59. doi:10.1038/nature16459. 
531 Vanparys, Bram, Eva Spieck, Kim Heylen, Lieven Wittebolle, Joke Geets, Nico Boon, and Paul De 532 Vos. 2007. "The Phylogeny of the Genus Nitrobacter Based on Comparative Rep-PCR, 16S

Wagner, Michael, Alexander Loy, Regina Nogueira, Ulrike Purkhold, Natuschka Lee, and Holger Daims. 2002. “Microbial Community Composition and Function in Wastewater Treatment Plants.” Antonie van Leeuwenhoek 81 (1-4): 665-80. doi:10.1023/a:1020586312170.

Whittaker, R H. 1965. “Dominance and Diversity in Land Plant Communities.” Science 147 (3655): 250 LP-260. http://science.sciencemag.org/content/147/3655/250.abstract.

Wright, Erik S. 2015. “DECIPHER: Harnessing Local Sequence Context to Improve Protein Multiple Sequence Alignment.” BMC Bioinformatics 16 (1). BMC Bioinformatics: 1-14. doi:10.1186/s12859-015-0749-z.

Wright, Erik S. 2016. “Using DECIPHER v2.0 to Analyze Big Biological Sequence Data in R.” The R Journal 8 (1): 352-59. doi:V12242009.

Xue, Kai, Liyou Wu, Ye Deng, Zhili He, Joy Van Nostrand, Philip G. Robertson, Thomas M. Schmidt, and Jizhong Zhou. 2013. “Functional Gene Differences in Soil Microbial Communities from Conventional, Low-Input, and Organic Farmlands.” Applied and Environmental Microbiology 79 (4): 1284-92. doi:10.1128/AEM.03393-12.

Zhou, Jizhong, Liyou Wu, Ye Deng, Xiaoyang Zhi, Yi-huei Jiang, Qichao Tu, Jianping Xie, Joy D Van Nostrand, Zhili He, and Yunfeng Yang. 2011. “Reproducibility and Quantitation of Amplicon 
551 Sequencing-Based Detection.” The ISME Journal 5 (8). Nature Publishing Group: 1303-13.

552 doi:10.1038/ismej.2011.11.

553 